\title{
CIX. Photometric investigation of the superficial resonance of sodium vapour
}

\section{Dunoyer \& R.W. Wood}

To cite this article: L. Dunoyer \& R.W. Wood (1914) CIX. Photometric investigation of the superficial resonance of sodium vapour , Philosophical Magazine Series 6, 27:162, 1025-1035, DOI: $10.1080 / 14786440608635172$

To link to this article: http://dx.doi.org/10.1080/14786440608635172

曲 Published online: 08 Apr 2009.

Submit your article to this journal $\square$

Џ Article views: 3

Q View related articles $\square$

4 Citing articles: 4 View citing articles ๘ 
No attempt was made to photograph the spectrum of the resonance radiation excited by $D_{1}$, as it is quite certain that, if the frequency of $D_{2}$ does not give rise to $D_{1}$, the same will hold true for $D_{1}$, as in all cases of resonance spectra the wave-lengths longer than those of the exciting light are much more intense than the shorter ones.

The mechanisms which produce the $\mathrm{D}_{1}$ lines are not, however, isolated completely, for it has been shown by one of us * that excitation of the vapour in the region of the channelled spectrum by means of blue-green light causes the appearance of the $\mathrm{D}$ lines in the emission spectrum, or at least of a yellow band which coincides with the position of the $\mathrm{D}$ lines. This band may, however, correspond to a curious band spectrum which is symmetrical about the $D$ lines which appeared in the spectrum excited by the cathode rays.

Laboratory of M. Bouty,

La Sorbonne, Paris.

UIX. Photometric Investigation of the Superficial Resonance of Sodium Vapour. By L. DuNoyer and R. W. Wood †.

[Plate XVII. figs. 8 \& 9.]

$7 \mathrm{TE}$ vapour of sodium, relatively cold, is capable of re1 emitting the $\mathrm{D}$ line radiations, when one concentrates on it light containing these same radiations. This was demonstrated by one of us $\ddagger$ in 1905 , the image of an oxyhydrogen sodium flame being formed by a large condenser along the axis of a highly exhausted tube containing a fragment of metallic sodium heated by a small Bunsen flame. At the same time it was shown that the cone of luminosity formed by the exciting rays retreated towards the wall, as the density of the vapour increased until there remained only a thin skin of yellow light, which lined the inner wall of the tube.

The experiment, as carried out at this time, was of short duration, and it did not appear possible to carry on any extensive investigations, with the disposition of the apparatus then employed.

The method of exciting the resonance has, however, recently been greatly improved by one of us $\$$ by using small glass bulbs, the walls of which are very carefully freed from occluded gases by prolonged heating, pure sodium being introduced into them by distillation. A further improvement consists in the employment of a Meker burner fed by

* Wood, Phil. Mag. x. p. 408 (1905).

$\dagger$ Communicated by the Authors.

$\ddagger$ Wood, Phil. Mag. x. p. 513 (1905).

\$ Dunoyer, Journal de Physique, iv. p. 17 (1914). 


\section{M. L. Dunoyer and Prof. Wood on Photometric}

the spray of a very dilnte solution of sodium chloride as a source of light, and forming an image of it on the wall of the bulb by an aplanatic condenser of very large aperture. The sharpness of this image permits of a study of the phenomenon of secondary resonance discovered by one of us in the case of the vapour of mercury and described recently ${ }^{*}$.

The surface of the bulb, illuminated in the manner described, becomes the source of a resonance radiation of remarkable brilliancy, of a thickness too small to be observed; as the duration of the phenomenon is ten or fifteen hours, it may be investigated photometrically or spectroscopically without difficulty. The preliminary study showed that the intensity of the resonance is much greater if a flame relatively poor in sodium is employed, than with a powerful flame such as is obtained if a fragment of salt is placed on the grill of a Meker burner. If the bulb is heated by a large flame coloured only by the sodium in the air of the room (previously charged by the operation of an intense sodaflame for a few minutes), one observes the yellow glow of the superficial resonance excited by the light cmitted by the flame used for the heating of the bulb. The flame must be waved about rapidly over the surface of the bulb, in order to secure a fairly uniform temperature. The phenomenon is less marked if an intense sodium flame is employed.

These experiments show that the greater part of the D light of the flame is inoperative in exciting the resonance. Moreover, the intensity of the source appears scarcely diminished if it is viewed through the bulb in which resonance is taking place. In other words, it is only the central cores of the $\dot{D}$ lines which are effective in exciting the resonance. The same phenomenon was observed in the study of the resonance of mercury vapour already alluded to, the luminosity (ultra-violet) excited by the 2536 line being enormously greater when the exciting mercury arc was first started, than after it had been in operation for a few seconds.

In the present communication we shall discuss :-

(1) The photometric study of the diffusing power of the highly attenuated vapour for monochromatic light, as compared with that of a white matt surface of magnesium oxide.

(2) The conditions under which all of the light removed from the exciting beam is re-emitted, giving us a diffuse reflecting power equal to that of the magnesium oxide.

* Wood, Phil. Mag. May 1912. 
Investigation of Superficial Resonance of Sodium Vapour. 1027

(3) The probable width of the spectrum lines emitted by the resonating vapour.

\section{Apparatus employed.}

The source of light for exciting the resonance was a Meker burner surrounded by an iron chimney perforated with a rectangular aperture. The burner was fed at the base with a spray formed by an atomizer operated by compressed air. A nearly saturated solntion ( 30 grs. to the litre) of $\mathrm{NaCl}$ was diluted to $\frac{1}{32}, \frac{1}{64}, \frac{1}{128}, \frac{1}{256}, \frac{1}{512}, \frac{1}{1024}$ and $\frac{1}{2048}$, and these solutions introduced in turn into the bulb of the atomizer, previously well rinsed out with a solution of the concentration employed.

An image of the window in the iron chimney was formed on the wall of the bulb by an aplanatic condenser of the type described recently by one of us ${ }^{*}$, having a diameter of 11 centimetres and a focus of $12 \mathrm{~cm}$. for parallel light. For divergent light, as in the present case, the source and image are each $25 \mathrm{~cm}$. from the lens.

As the sodium bulbs used in these experiments are very easily made, and are extremely convenient for illustrating

Fig. 1.

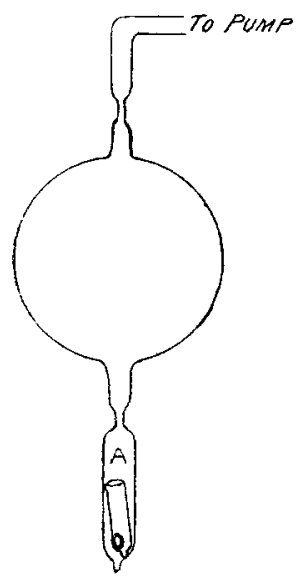
resonance radiation, it may be well to devote a few words to the manner of preparing them. The bulbs are $5 \mathrm{~cm}$. in diameter, drawn down to $1 \mathrm{~mm}$. capillaries as shown in fig. 1 . The sodium must first be heated to fusion in a small test-tube and poured out on a cool surface. A piece about $2 \times 2 \times 2$ mm. is introduced into a small piece of very thin-walled glass tubing, closed at one end, and this capsule placed in the lower tube A, which serves as the distillation chamber. The lower end of $A$ is now closed in the flame of a blastlamp, and the tube sealed to the mercury pump. After exhausting to a pressure of $001 \mathrm{~mm}$. the bulb is heated for four or five minutes with a large Bunsen flame, the pump working all the while. It should be heated as hot as possible without collapsing. After the bulb has cooled off, the flame is carefully applied to the chamber $\mathbf{A}$, and the sodium distilled into

- Dunoyer, Journal de Physique, iii. p. 468 (1913). 


\section{M. L. Dunoyer and Prof. Wood on Photometric}

the bulb. The lower capillary is then sealed, and finally the upper. The pump should be working vigorously all the while, as the brilliancy of the resonance depends upon having the highest possible vacuum. In our experiments we beated the bulb for twenty minutes, to make sure of getting rid of all of the gases, and the sodium was previously heated in cacuo, but these extreme precautions are not necessary in the preparation of bulbs for lecture purposes.

The bulb was supported by a wire in a column of hot air rising from a large tube of fireciay with a large Meker burner at the bottom, by means of which a fairly uniform temperature up to $400^{\circ}$ could be obtained. For lecture purposes it is sufficient to heat the bulb by waving a large Bunsen flame over its entire surface. The arrangement of our apparatus is shown in fig. 2. As a standard source of

Fig. 2.

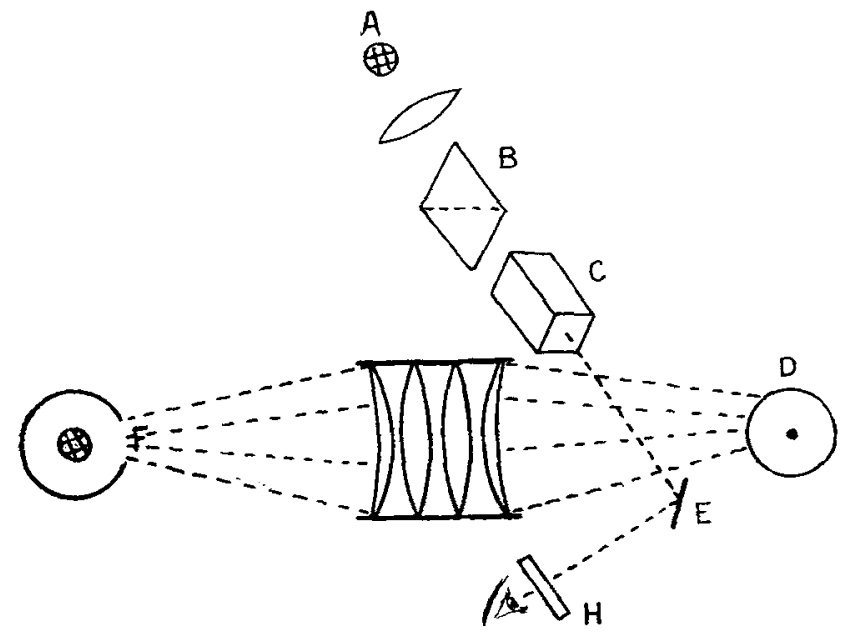

comparison we used a sodium flame $A$ behind a piece of ground glass mounted behind a pair of large Nicol prisms $B$ and $C$. The sodium bulb $D$ was first coated with magnesium oxide, by holding it above a piece of burning $\mathrm{Mg}$ ribbon. It was then wiped clean, a small square patch of the oxide being left on one side. The image of the window in the iron chimney surrounding the exciting sodium flame $F$ was formed upon the square of magnesia and the adjacent clear glass of the bulb. It was our object to measure the ratio of the intensity of the magnesia and the vapour of sodium under equal illumination. This was done by means of a very 
simple photometer which consisted of a thin scale of silvered glass $\mathrm{E}$, with a razor edge, made by silvering a piece of plate glass, polishing it, and then striking the edge with a hammer. 'This mirror reflected the comparison source $A$ to the eye through a cell $H$ containing a solution of bichromate of potash (to remove the green and blue rays of the Bunsen flame). Behind the sharp edge of the silver mirror the illuminated surfaces of magnesia and sodium vapour could be seen at the same time, and by adjusting the Nicol prism C: the edge of the mirror could be made to disappear, first when seen against the magnesia and secondly against the background of resonating vapour. The intensities of the two surfaces are then in the ratio of the squares of the angles through which $\mathrm{C}$ is turned from the position of extinction. The temperature to which the bulb was heated by the ascending current of hot air was about $330^{\circ}$, measured with a nitrogen mercury thermometer.

The results are given in the following table, the concentrations of the salt solution in the atomizer bulb in the first column, the angles of the Nicol prism $\mathrm{C}$ in the next two columns, and the ratio in the fourth.

\begin{tabular}{|c|c|c|c|}
\hline $\begin{array}{c}\text { Solution } \\
\text { Concentration. }\end{array}$ & $\begin{array}{c}\text { Angle } a \text { of Nicol } \\
\text { for Resonance } \\
\text { Radiation. }\end{array}$ & $\begin{array}{l}\text { Angle } a^{\prime} \text { of Nicol } \\
\text { for Magnesia. }\end{array}$ & $\begin{array}{c}\text { Ratio } \frac{\sin ^{2} n^{\prime}}{\sin ^{2} \alpha} \\
\text { I' }^{\prime} \text { (Magnesia) } \\
\text { I Sodium vapour }\end{array}$ \\
\hline$\frac{1}{2048}$ & $3^{\circ}$ & $6^{\circ}$ & 4 \\
\hline$\frac{1}{1024}$ & $9^{\circ} \cdot 25$ & $19^{\circ}$ & 4.5 \\
\hline$\frac{1}{512}$ & $10^{\circ} \cdot 5$ & $22 \circ \cdot 5$ & $4 \cdot 8$ \\
\hline$\frac{1}{256}$ & $13^{\circ} \cdot 5$ & $36^{\circ} \cdot 4$ & $6 \cdot 3$ \\
\hline$\frac{1}{128}$ & $14^{0.4}$ & $45^{\circ}$ & $9 \cdot 6$ \\
\hline$\frac{1}{64}$ & $14^{\circ}$ & $67^{\circ}$ & 15 \\
\hline$\frac{1}{32}$ & 1206 & $90^{\circ}$ & 19 \\
\hline
\end{tabular}

We see from this table that even with the most dilute solution the diffuse reflecting power of the magnesia is four Phil. Mag. S. 6. Vol. 27. No. 162. June 1914. 3 Y 


\section{M. L. Dunoyer and Prof. Wood on Photometric}

times as great as that of the resonating sodium vapour, for the total yellow light of the flame. This is of course due to the circuinstance that the magnesia reflects all of the D light, while the vapour scatters only the light corresponding to the cores of lines, the light of the edges of the lines being transmitted. As the concentration increases the intensity of the resonance radiation increases but slightly after a certain point is reached. since the gain in the intensity of the sodium flame then results chiefly from a widening of the lines. For the most concentrated solution $\left(\frac{1}{32}\right)$ the magnesia was 19 times as intense as the vapour. On reducing the aircurrent until the yellow colour of the flame was barely visible, a ratio of 3 was obtained, the values of the angles being $2^{\circ}$ and $3^{\circ} .5$. This result was, however, open to question on account of the faintness of the light.

The above results are in accord with those previously obtained by one of us by a different method *.

If now the molecular resonators absorb none of the light which they abstract from the exciting beam, we ought, if the exciting radiations are made sufficiently homogeneous, to have all of the light diffusely reflected by the vapour; in other words, our ratio ought to sink to unity when the D lines in the source become infinitely narrow. It is impossible to reach this point by diminishing the amount of sodium in the flame, our lowest value for the ratio being four, or perhaps three.

We have, however, investigated the matter by employing the principle of the resonance lamp previously described by one of us, which has been used in the investigations on the resonance of mercury vapour. The experiment was made by utilizing the spot of superficial resonance as a source of light for exciting the vapour at a different point on the surface of the bulb. The arrangement of the apparatus is shown in fig. 3. A small triangular spot of magnesia was formed on the surface of the sodium bulb with a black dot of lamp-black at its centre to indicate its position. The image of the sodium flame was thrown upon this spot, and the magnesia triangle shone brilliantly upon the less intense background of the resonance radiation (fig. 3,a). The bulb was now rotated until the triangle of magnesia was in darkness, and an image of the spot of resonance radiation thrown upon it by means of a large concave mirror, formed by

* L. Dunoyer, Joumal de Physique, iv. p. 17 (1914). 
Investigation of Superficial Resonance of Sodium Fapour. 1031 Fig. 3.

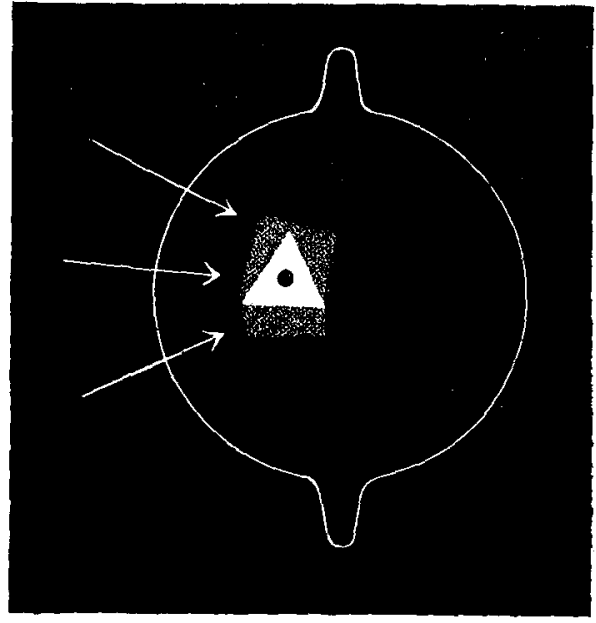

$a$.

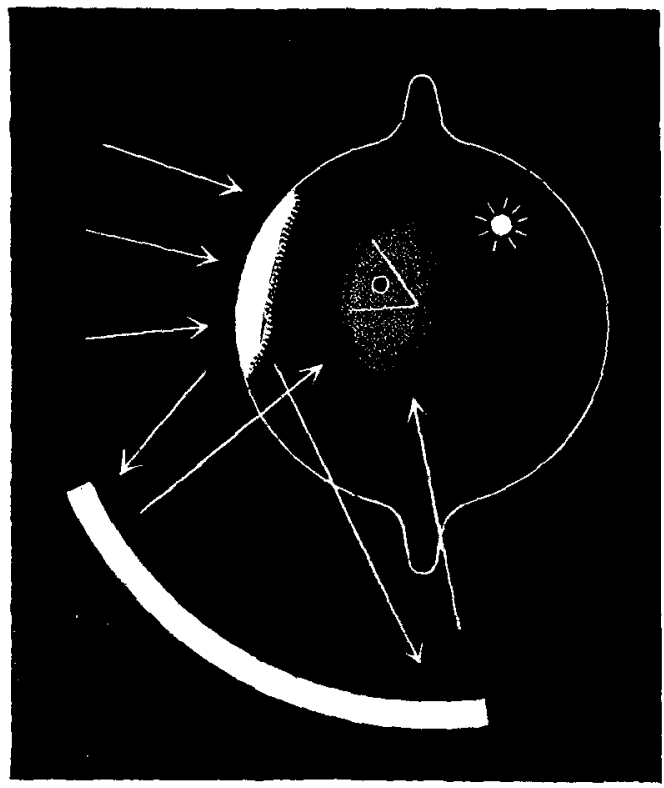

$b$. 


\section{M. I. Dunoyer and Prof. Wood on Photometric}

silvering one surface of a double convex lens. Under these conditions the magnesia triangle, and the resonance radiation (which may be termed secondary) which surrounded it had practically the same intensity. In fact it was only with difficulty that the outline of the triangle could be seen, the black dot being surrounded by a uniform glow of light of oval outline (fig. $3, b$ ).

Photographs taken of the phenomena are reproduced on Pl. XVII. figs. 8 and 9 , the latter showing the disappearance of the magnesia triangle, when the area is illuminated by resonance light reflected from the mirror. The brilliantly illuminated area to the left is the primary resonance excited by the rays from the flame. A narrow dark line partially outlines the triangle; this is due to the shadow thrown upon the resonating vapour by the edges of the layer of magnesia.

The complete disappearance of the triangle was observed only when the flame for exciting the primary resonance was very poor in sodium. We thus have a ratio equal to unity when the exciting rays are sufficiently homogeneous, and can safely say that no true absorption exists in the case of sodium vapour at very low density and in a high vacuum, though the spectroscope would of course show an absorption line. All of the energy abstracted from the primary beam is re-emitted by the molecules, precisely as was found for mercury vapour.

\section{Probable width of the resonance lines.}

The experiments which we have just described show that the resonance radiation of sodium is excited by the narrow central regions of the $D$ lines.

Let $A B C$ of fig. 4 represent the intensity curve of one of the exciting lines, and the dotted curve DBE the region effective in exciting resonance. The intensity curve of the emitted resonance radiation will be of similar dimensions and may be represented by $\mathrm{F}$. The ratio of the area of the curve $\mathrm{ABC}$ to the area of $\mathrm{DBE}$ is obviously the ratio found by the photometric measurements, and if we know the form of the curves, and the actual dimensions of $\mathrm{ABC}(i . e$. the width of the line in the flame spectrum), we can, from our experimentally found ratio of $4: 1$, determine the width of $\mathrm{DBE}$, the line of the resonance radiation.

The interferential measurements of Fabry and Buisson have shown that the widths of the $D$ lines emitted by a flame poor in sodium are $0.08 \AA$. 
Investigation of Superficial Resonance of Sodium Vapour. 1033

The law of the partition of energy in spectrum lines furnished by the kinetic theory of gases is

$$
y=\mathrm{C} e^{-k x^{2}}
$$

in which $y$ is the intensity at a distance equal to $x$ from the centre of the line (at which point the intensity is C), and $k$ gives us the rate at which the intensity falls off as we depart from the centre.

Fig. 4.

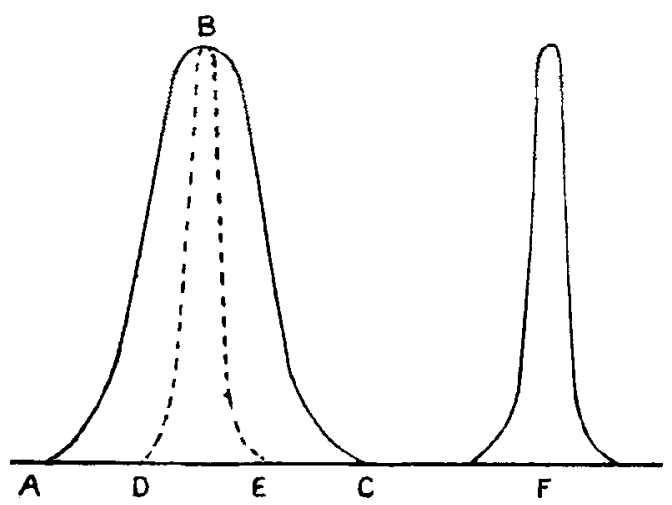

The width $2 \epsilon$ of a spectrum line, as defined by Fabry and Buisson, is the distance between two ordinates at distances $\epsilon$ from the centre, of value equal to $\frac{\mathrm{C}}{2}, i_{\text {. }}$ e. an intensity onehalf as great as that at the centre of the line.

This gives us

$$
\begin{aligned}
& \frac{1}{2}=\frac{\mathrm{C} e^{-k \epsilon}}{\mathrm{C}} \text { and } e^{-k \epsilon}=2, \text { or } \\
& \sqrt{k} \epsilon=\sqrt{\log 2} . . \quad . \quad .
\end{aligned}
$$

The total intensity of the line being the area comprised between the curve and the axis of abscissa, we have

$$
\mathrm{I}=\int_{0}^{\infty} \mathrm{C} e^{-k x^{2}} d x=\frac{\mathrm{C} \sqrt{\pi}}{\sqrt{k}} \cdot . \cdot \cdot
$$

If we assume that the portion of the exciting line effective in exciting the resonance is comprised between the ordinates situated at $-x_{1}$ and $+x_{1}$ (which is of course an approximation since in reality the exciting portion of the line is as shown by the dotted curve of fig. 4), we have, for the 
intensity removed from the line by the resonating molecules,

or for the ratio

$$
\mathrm{I}^{\prime}=\int_{-x_{1}}^{+x_{1}} \mathrm{C} e^{-k x} d x=\frac{2 \mathrm{C}}{\sqrt{k}} \int_{0}^{\sqrt{k} x_{1}} e^{-u^{2}} d u,
$$

$$
\frac{\mathbf{I}^{\prime}}{\mathrm{I}}=\frac{2}{\sqrt{ } \pi_{2}} \int_{0}^{\sqrt{\bar{k}} x_{2}} e^{-u^{2}} d u
$$

Now the value of $\frac{I^{\prime}}{\mathrm{I}}$ has been determined by the photometric experiments. For the concentration $\frac{1}{2048}$, or the flame containing the least amount of sodium $\frac{\mathrm{I}^{\prime}}{\mathrm{I}}=0 \cdot 25$.

From this value and the tables of integrals (Calculus of Probabilities of M. Bachlier) we can calculate the value of the upper limit

$$
\sqrt{k} x_{1}=0.0225 ; \quad \text {. . . . . }
$$

and by division (equations 2 and 4 )

$$
\frac{x_{1}}{\epsilon}=0.27 . . \cdot \text {. . . . . }
$$

We may obtain an approximate value of $x_{1}$ if we take for $\epsilon$ the value given for a feeble sodium flame by Fabry and Buisson,

$$
\epsilon=0 \cdot 04 \AA \text {. }
$$

Inserting this value in equation (5) gives us

$$
x_{1}=0.0108 \AA \text {, }
$$

or, since $x_{1}$ is the half width of the region required, for the width of the region effective in exciting resonance,

$$
2 x_{1}=\cdot 021 \AA \text {, }
$$

the probable width of the resonance lines in contrast to

$$
2 \epsilon=\cdot 08 \AA \text {, }
$$

the width of the flame lines.

Wo thus see that by means of sodium vapour at low temperature we can manufacture, so to speak, light much more homogeneous than the incident light, the method being somewhat analogous to that of the Residual Rays of Rubens and Nichols.

It is highly probable that the width of the region removed from the exciting line is identical with the width of the re-emitted resonance radiation. The lines obtained in this 
way are thus only one quarter of the width of the lines emitted by the flame and narrower than the iron are lines.

They are, however, three times as wide as the narrowest known line, the red line of cadmium, for which

$$
2 \epsilon=\cdot 006 \AA \text {. }
$$

An interferometer study of the resonance radiation is much to be desired, for the above method of deducing the width of the lines is somewhat circuitous.

Laboratory of M. Bouty,

La Sorbonne, Paris.

\section{Proceedings of Learned Societies.}

GEOLOGICAL SOCIETY.

[Continued from p. 540.]

January 21st, 191 t.-Dr. Aubrey Strahan, F.R.S., President, in the Chair.

THE following communications were read:-

1. 'Geology of the Country round Huntly (Aberdeenshire). By William Robert Watt, M.A., B.Sc., F.G.S.

In this area two distinct series of rocks can be distinguished-a foliated, and a non-foliated series. In the former occur rocks originally sedimentary and others originally igneous. In the nonfoliated series, which is wholly of igneous origin, three main intrusions occur :-(1) The earliest and most extensive is a norite with, as modifications due to differentiation, olivine-gabbros, troctolites and 'picrites.'

(2) Into this is intruded the heterogeneous mass known as the Central Intrusion, which consists of three main types with no distinct boundaries :-

(a) At the margin occurs a fine-grained norite with pronounced mineral banding. Nearer the centre of the mass is met $(b)$ a biotite-plagioclase rock; and the centre itself is composed of (c) a garnet-monzonite.

(3) The third large intrusion is the Carvichen Granitite, composed chiefly of quartz, microcline, and biotite.

Each of these masses produces some contact-alteration in the surrounding foliated or non-foliated rocks. Where the Central Intrusion or the Carvichen Granitite is intruded into the earlier norite, a norite containing cordierite is produced. The original morite, by absorption of sediment, produces also along its margin a cordierite-norite. Similar types have been described by Prof. A. Lacroix and Mr. A. N. Winchell.

Both types of cordierite-norite tend to pass into a rock composed essentially of plagioclase, biotite, and garnet; and this change, with the gradual destruction of the hypersthene, can be seen in various stages. 
WOOD \& DUxoyer.

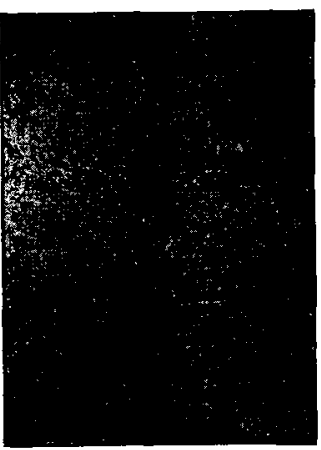

Fig. 1.

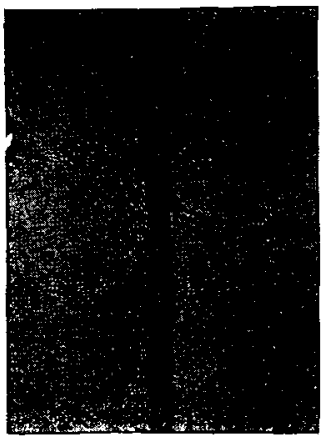

FIલ. 3.

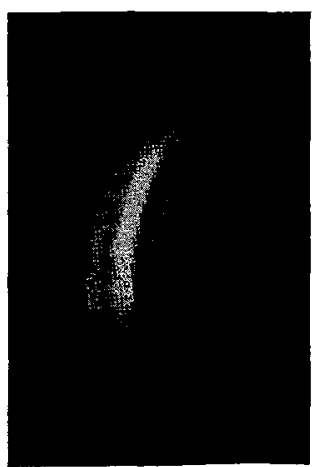

Fig. 5.
Phil. Mag. Ser. 6, Vol. 27, Pi. XVlI.

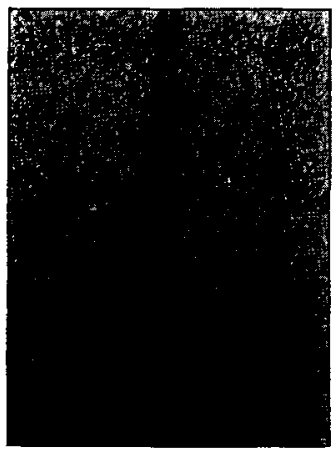

FIG. 2.

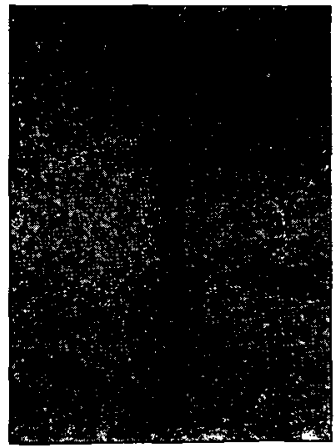

FIG. 4.

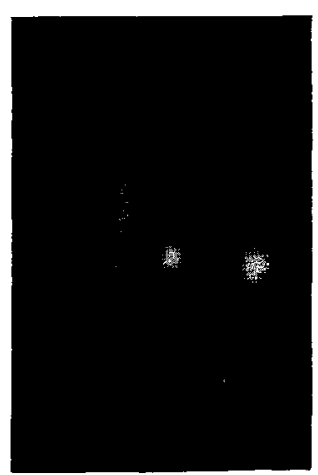

FIG. 6.

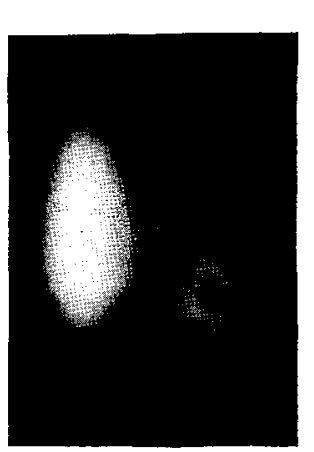

FIG. 9.

FIG. 8.
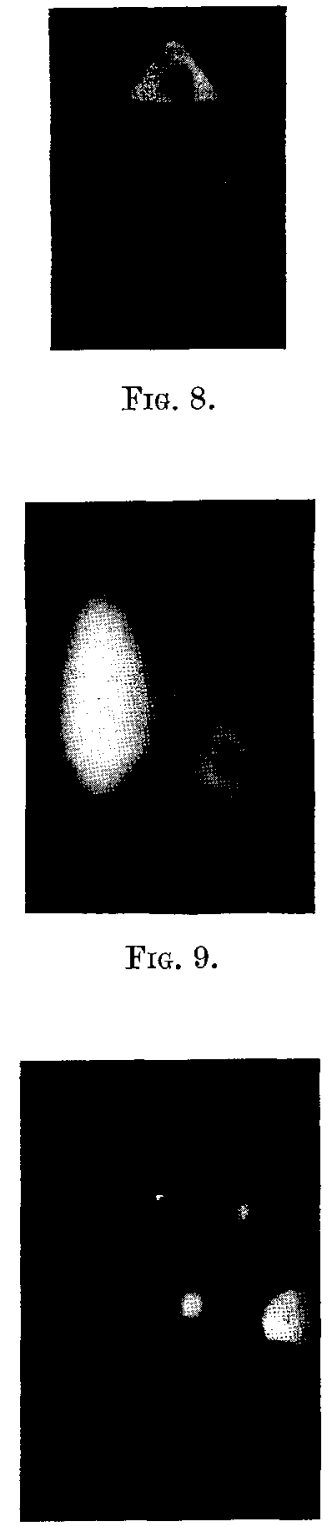

FIG. 7. 